\title{
COMPORTAMENTO DA ATOMIZAÇÃO ELETROTÉRMICA DE OURO, PRATA, BISMUTO, CÁDMIO, CHUMBO E ESTANHO EM SOLUÇÕES AQUOSAS E EM ETANOL, A PARTIR DE DIFERENTES SUPERFÍCIES ATOMIZADORAS
}

\author{
José Bento Borba da Silva \\ Departamento de Química, Universidade Federal de Minas Gerais, CP 702, 31270-901 Belo Horizonte - MG
}

Recebido em 11/3/03; aceito em 9/1/04; publicado na web em 27/05/04

\begin{abstract}
BEHAVIOR OF ELECTROTHERMAL ATOMIZATION OF GOLD, SILVER, BISMUTH, CADMIUM, LEAD, AND TIN IN AQUEOUS SOLUTIONS AND IN ETHANOL STARTING FROM DIFFERENT ATOMIZING SURFACES. The atomization behavior of $\mathrm{Au}, \mathrm{Ag}, \mathrm{Bi}, \mathrm{Cd}, \mathrm{Pb}$, and $\mathrm{Sn}$ from pyrolitic graphite coating (L'vov platform) with the use Pd and $\mathrm{Mg}$ solutions, and zirconium coated platform with the analytes in nitric acid $0.2 \% \mathrm{v} / \mathrm{v}$ and in ethanol was investigated. In ethanol medium, the sensitivity gain was three-fold for $\mathrm{Bi}$ and $\mathrm{Cd}$ using $\mathrm{Zr}$ as modifier. Without modifier, the ethanol medium is appropriate only for $\mathrm{Au}$ and $\mathrm{Cd}$. In nitric acid medium, the $\mathrm{Zr}$ coated platform elevates sensitivity at least two-fold for $\mathrm{Bi}$ and $\mathrm{Cd}$. The method was applied to the determination of $\mathrm{Ag}, \mathrm{Au}$ and $\mathrm{Bi}$ of certified steel samples, after on-line preconcentration, sorption on a minicolumn filled with $\mathrm{C}-18$ bonded to silica gel and elution with ethanol. The concentrations obtained agreed with the recommended values.
\end{abstract}

Keywords: atomization behavior; electrothermal atomic absorption spectrometry; zirconium permanent.

\section{INTRODUÇÃO}

Determinações de diferentes metais empregando a técnica de espectrometria de absorção atômica com atomização eletrotérmica em forno de grafite (ET AAS) têm sido objeto de um número de estudos, principalmente considerando que, para cada elemento em particular, as condições mais favoráveis para a obtenção de determinações mais exatas, precisas e com boa sensibilidade, as quais incluem otimizações do programa de temperatura do forno, bem como da natureza e concentração das substâncias empregadas para modificação química, devem ser otimizadas para cada matriz e analito em particular.

De forma mais geral, o emprego de ET AAS ganhou impulso pela sensibilidade e possibilidade que a técnica oferece de pré-separação dos concomitantes da matriz na etapa da pirólise. Desde a introdução do conceito STPF ("Stablized Temperature Plataform Furnace") ${ }^{1}$, que envolve entre outras condições o uso de modificadores químicos ${ }^{2}$, o comportamento de diferentes substâncias passíveis de atuarem como modificadores químicos tem sido estudado para um número de metais em diferentes matrizes. O emprego de modificadores químicos tem sido considerado como parte essencial nas determinações por ET AAS, com função de aumentar a volatilidade da matriz permitindo, assim, sua separação nas etapas preliminares do programa de temperatura do forno, ou como substância(s) que $\operatorname{possa}(\mathrm{m})$ permitir uma estabilização térmica do analito em temperaturas mais elevadas ${ }^{3-6}$.

A mistura de Pd com Mg tem sido empregada como modificador químico universal, pela sua capacidade de estabilizar a maior parte dos elementos determinados por $\mathrm{ET} \mathrm{AAS}^{3-5}$, constando como recomendação em programas de manuais de fabricantes de equipamentos ${ }^{7}$. Por outro lado, outros elementos químicos passíveis de atuarem como modificadores têm sido descritos. Neste sentido, pode ser destacado o uso de elementos do grupo da platina (Pt, Pd, Ir, Rh e Ru ${ }^{6,8-24}$, bem como do uso de elementos formadores de carbetos metálicos $(\mathrm{Zr}, \mathrm{Nb}, \mathrm{Ta}, \mathrm{W})^{25-35}$.

Quando o elemento empregado como modificador químico é aplicado anteriormente à etapa da determinação, isto é, quando este é

*e-mail: bentojb@terra.com.br aplicado sobre o tubo de grafite ou sobre uma plataforma de L'vov e submetido a tratamento térmico de forma a possibilitar a obtenção de uma superfície modificada na plataforma ou diretamente no tubo, obtém-se o que se convencionou chamar de modificador químico permanente. Entre os exemplos deste tipo de atuação do modificador podem se destacar a aplicação de $\mathrm{Ir}^{6,9,11,14,17,18}, \mathrm{Rh}^{11,13,14,17,18}$, mistura de Ir e $\mathrm{Rh}^{18,21}, \mathrm{Ru}^{14,16,19,21-24}$, além dos elementos formadores de carbetos como $\mathrm{Zr}$ e $\mathrm{W}^{14-21,31,33}$, de misturas de $\mathrm{W}$ com $\mathrm{Rh}^{34,35}$, e de $\mathrm{Zr}$ com outros metais nobres ${ }^{27,28,30-34}$.

De acordo com uma revisão efetuada por Tsalev et al. ${ }^{25}$, o emprego de superfícies (parede do tubo de grafite ou plataforma inserida neste) pré-tratadas com elementos do grupo da platina ou daqueles formadores de carbetos, tem sido apontado como uma alternativa para determinações mais rápidas, com possibilidade de purificação do modificador durante a etapa de deposição deste ${ }^{18-20}$ possibilitando, assim, a obtenção de brancos mais baixos e consequiente melhora nos limites de detecção ${ }^{34,35}$, bem como de determinações mais rápidas e exatas. Por outro lado, atomização a partir destas superfícies, pode propiciar também melhor separação da matriz na etapa de pirólise e a obtenção do analito em uma forma química apropriada na etapa da atomização. De acordo com Tsalev et al. ${ }^{25}$, os efeitos destas superfícies modificadoras podem estar associados ou a um comportamento muito similar ou distinto entre analito e modificador, ou seja, no primeiro caso, um isomorfismo entre o analito e estes elementos modificadores pode favorecer a retenção do analito mais fortemente no interior da rede cristalina do cristal do modificador. No caso de dissimilaridade, esta pode favorecer a formação de forte ligação química entre ambos ${ }^{25}$.

As determinações de metais efetuadas por ET AAS, em sua grande maioria, são obtidas com o analito adequadamente solubilizado em uma solução aquosa levemente ácida, geralmente em ácido nítrico diluído $(0,2 \% \mathrm{v} / \mathrm{v})^{7,8,18,34}$. Por outro lado, em algumas situações pode ser conveniente que a solubilização do analito seja efetuada em soluções orgânicas, notadamente em etanol. Em um número de procedimentos propostos para pré-concentração de diferentes analitos, especialmente envolvendo sistemas em fluxo, o etanol tem se destacado como um bom eluente, por uma série de características favoráveis comparativa- 
mente a outras substâncias empregadas com este propósito. Isso é verdadeiro e tem sido aplicado em diferentes sistemas propostos empregando diferentes diagramas FIA e agentes complexantes. Diversos autores têm citado o etanol com um dos mais apropriados eluentes para este tipo de sistemas de complexação, seja empregando sílica modificada com grupamentos funcionais octadecil (RP-Si-C ${ }_{18}$ ) como adsorvente ${ }^{36-41}$, reatores feitos de PTFE ${ }^{42-45}$, amberlite XAD-16 ${ }^{46}$, bem como em complexos retidos em mini-coluna preenchida com carvão ativo $^{47}$. Outros sistemas que empregam etanol associado a outras substâncias como meio químico para o analito têm sido descritos ${ }^{48}$. Tem-se observado que são diferentes as sensibilidades dos elementos quando etanol é empregado em lugar de soluções fracamente ácidas. Neste sentido, para determinação de chumbo em vinhos, Matheus e Parsons ${ }^{49}$ observaram a necessidade de efetuar ajuste de matriz nas soluções analíticas empregadas para calibração com uma quantidade média de etanol contida nos vinhos para não haver perdas na exatidão das determinações. Por outro lado, em determinações por ET AAS empregando a introdução das amostras na forma de suspensões, alguns autores têm enfatizado vantagens no emprego de misturas das quais um dos componentes utilizado, em diferentes concentrações, é o etanol ${ }^{50-58}$.

Não obstante o crescente uso de etanol como um meio apropriado para preparação do meio químico do analito, são escassos os estudos efetuados em relação ao comportamento de atomização de metais freqüentemente determinados por ET AAS em meio etanol, bem como do uso apropriado de modificadores químicos neste meio químico.

No presente estudo foram averiguados os comportamentos de um grupo de elementos determinados por ET AAS (Au, Ag, Bi, Cd, $\mathrm{Pb}$ e $\mathrm{Sn}$ ), tanto em meio ácido como em etanol, em relação ao não uso de modificador químico, ao uso de mistura de Pd e Mg e com uso de superfícies (plataforma de L'vov) recobertas com zircônio. Foram investigados e comparados através de curvas de temperaturas de pirólise e de atomização as temperaturas adequadas em cada etapa.

\section{PARTE EXPERIMENTAL}

\section{Equipamentos}

As medidas de absorvância integrada foram conduzidas em um espectrômetro de absorção atômica Perkin Elmer AAnalyst 100 (Norwalk, CT, EUA), equipado com forno de grafite HGA-800 e amostrador automático AS-72, com corretor de fundo por lâmpada de arco de deutério. As condições operacionais foram aquelas sugeridas pelo fabricante do equipamento e estão resumidas na Tabela 1. As lâmpadas usadas foram mono-elementares de catodo-oco provenientes da Hitachi (Mitorika, Ibaraki, Japão) para Au (Part No 139-3581), Ag (Part. No. 139-3614) e Bi (Part. No 139-3564), e da Perkin-Elmer para Cd (Part. No N3050-115), Pb (Part. No. N3050-117) e Sn (Part No. N3050-175). O volume de soluções analíticas introduzido no forno foi de $20 \mu \mathrm{L}$. Quando um modificador químico na forma de solução (mistura de Pd e Mg, em massas de 15 e $10 \mu \mathrm{g}$, respectivamente) foi empregado junto com as soluções analíticas (tanto em meio de ácido nítrico $0,2 \% \mathrm{v} / \mathrm{v}$, como em meio de etanol), o volume deste foi de $10 \mu \mathrm{L}$. Argônio (99,996\%, White Martins, Belo Horizonte - MG, Brasil) foi usado como gás de purga. Para todos os analitos, foram empregados tubos de grafite de revestimento pirolítico (Perkin-Elmer Part No. B010-9322) contendo plataformas de L'vov convencionais totalmente pirolíticas (Perkin-Elmer Part No. B010-9324).

\section{Reagentes}

Todos os reagentes empregados foram de grau analítico ou de elevada pureza. A água foi deionizada em sistema do tipo Milli-Q (resistividade inferior a 18,2 M $\Omega \mathrm{cm}$ - Millipore, Bedford, MA, USA). O ácido nítrico (Carlo Erba, Milão, Itália, No.408015) foi purificado por destilação abaixo do seu ponto de ebulição em destilador de quartzo (Kurner Analysentechnik, Rosenheim, Alemanha). O etanol empregado foi da Carlo Erba (No. 414629).

As seguintes soluções estoque (1000 $\left.\mathrm{mg} \mathrm{L}^{-1}\right)$ foram empregadas: Zircônio - Aldrich (Milwaukee, WI, EUA, No. 27497-6) em 5\% v/v de $\mathrm{HCl}$;

Bismuto - Aldrich (No. 20699-7) em 5\% v/v de $\mathrm{HNO}_{3}$;

Cádmio - Merck (Darmstadt, Alemanha, No. 102597), a 1,0 mol em $\mathrm{HCl}$;

Chumbo - Spex (Edison, N.J., EUA, PLK10-Pb) a 0,3 mol L-1 em

$\mathrm{HCl}$;

Estanho -Spex (PLK10-Sn) a $1 \mathrm{~mol} \mathrm{~L}^{-1} \mathrm{em} \mathrm{HCl}$;

Ouro - Aldrich (No. 20716-0) a 0,5 mol L-1 em $\mathrm{HNO}_{3}$;

Prata - Fluka (Buchs, Suíça, No. 85137) a 0,5 mol L-1 em $\mathrm{HNO}_{3}$.

Para os modificadores em solução os seguintes reagentes $\left(10.0 \pm \mathrm{mg} \mathrm{L}^{-1}\right)$ foram empregados:

Nitrato de magnésio, modificador para ET AAS, (Merck No. B593213 431) e

Nitrato de paládio, modificador para ET AAS, (Merck No. B9366989 710).

Antes do uso, toda a vidraria foi lavada abundantemente com uma solução de detergente (Extran, Merck, Art. 7553) e enxaguadas com água morna. Subseqüentemente, foram mantidas em solução de ácido nítrico $50 \%$ v/v por, no mínimo, $48 \mathrm{~h}$ e posteriormente lavadas 6 vezes com água deionizada. Os copos de PTFE do amostrador automático do ET AAS foram limpos, sendo igualmente submetidos ao mesmo tratamento.

\section{Procedimento}

Os primeiros estudos foram efetuados empregando um tubo novo com plataforma de L'vov para a obtenção das curvas de temperatura de pirólise e de atomização na ausência de modificador químico, para cada um dos analitos em soluções preparadas em ácido nítrico $0,2 \% \mathrm{v} / \mathrm{v}$. Um outro tubo novo foi empregado para obtenção das curvas de temperatura de pirólise e de atomização para os elementos solubilizados em etanol.

Em uma segunda etapa, foram obtidas curvas de temperatura de pirólise e de atomização para este mesmo conjunto de metais, empregando solução de Pd e Mg como modificador químico (15 e 10 $\mu \mathrm{g}$, respectivamente) $)^{7}$, em outro tubo novo para soluções em ácido nítrico $0,2 \% \mathrm{v} / \mathrm{v}$ e em outro tubo-plataforma para soluções preparadas em etanol.

Tabela 1. Parâmetros instrumentais e condições operacionais para ET AAS

\begin{tabular}{lcccccc}
\hline Parâmetro & $\mathrm{Ag}$ & $\mathrm{Au}$ & $\mathrm{Bi}$ & $\mathrm{Cd}$ & $\mathrm{Pb}$ & $\mathrm{Sn}$ \\
\hline Comprimento de onda (nm) & 328,1 & 248,8 & 222,8 & 228,8 & 283,3 & 224,6 \\
Resolução espectral (nm) & 0,7 & 0,7 & 0,2 & 0,7 & 0,7 & 0,7 \\
Corrente da lâmpada (mA)* & 13 & 15 & 13 & 6 & 10 & 25 \\
\hline
\end{tabular}

* Lâmpadas monoelementares de catodo-oco. 
Para averiguar o comportamento da camada de zircônio como modificador químico permanente para cada um destes metais, a plataforma tratada com o zircônio foi obtida, de maneira semelhante à empregada anteriormente ${ }^{18,19}$, i. e., aplicando-se o programa de temperatura de forno (Tabela 2) a alíquotas de $40 \mu \mathrm{L}$ de solução de zircônio $\left(500 \mu \mathrm{g} \mathrm{L}^{-1}\right)$, repetindo-se por 25 vezes, de modo a obter uma camada de $500 \mu \mathrm{g}$ de zircônio sobre a plataforma. Após obtida a cobertura da plataforma, conforme pode-se observar pela Tabela 2, foram efetuadas duas etapas adicionais, com o objetivo de transformar os óxidos e/ou oxicarbetos de zircônio em carbetos do metal ${ }^{6,34,35}$. Estas etapas foram empregadas anteriormente em plataformas recobertas com $\mathrm{W}^{34,35} \mathrm{e}$, considerando-se as características similares citadas na literatura ${ }^{9,59}$ nas espécies químicas formadas no interior do forno por estes dois elementos, estes passos foram aqui aplicados para depositar carbeto de zircônio sobre a plataforma.

O programa apresentado na Tabela 3 foi empregado para a obtenção dos sinais analíticos para todos os metais em estudo nos dois meios químicos. O programa descrito na Tabela 4, por sua vez, foi empregado no estudo da influência da massa de $\mathrm{Zr}$ depositada sobre a plataforma nas sensibilidades observadas para $\mathrm{Bi}, \mathrm{Cd}$ e $\mathrm{Sn}$.

Tabela 2. Sequência para cobertura da plataforma de L'vov com $\mathrm{Zr}$ $(500 \mu \mathrm{g})$

\begin{tabular}{|c|c|}
\hline Etapa & Ações e parâmetros \\
\hline 1 & $\begin{array}{l}\text { Pipetar } 40 \mu \mathrm{L} \text { de solução de } \mathrm{Zr}\left(500 \mu \mathrm{g} \mathrm{L}^{-1}\right) \text { sobre a } \\
\text { plataforma }\end{array}$ \\
\hline 2 & $\begin{array}{l}\text { Aplicar o seguinte programa de aquecimento } \text { (rampa, }^{\mathrm{a}} \text {, } \\
\text { tempo de permanência) para secagem e pirólise e limpeza } \\
\text { do modificador: } 90^{\circ} \mathrm{C}(5,15 \mathrm{~s}), 140{ }^{\circ} \mathrm{C}(5,15 \mathrm{~s}), 1000^{\circ} \mathrm{C} \\
(10,10 \mathrm{~s}), 2000^{\circ} \mathrm{C}(0,5 \mathrm{~s})\end{array}$ \\
\hline 3 & $\begin{array}{l}\text { Repetir as etapas } 1 \text { e } 2 \text { por mais } 24 \text { vezes, de forma a } \\
\text { obter uma camada com } 500 \mu \mathrm{g} \text { de } \mathrm{Zr} \text { sobre a plataforma }\end{array}$ \\
\hline 4 & $\begin{array}{l}\text { Aplicar quatro vezes o programa de temperatura (rampa, } \\
\text { tempo de aquecimento) para condicionamento do carbeto } \\
\text { de zircônio à temperatura moderada }: 150{ }^{\circ} \mathrm{C}(1,10 \mathrm{~s}) \text {, } \\
600{ }^{\circ} \mathrm{C}(10,15 \mathrm{~s}), 1100{ }^{\circ} \mathrm{C}(10,5 \mathrm{~s}), 1400{ }^{\circ} \mathrm{C}(10,10 \mathrm{~s})\end{array}$ \\
\hline 5 & $\begin{array}{l}\text { Aplicar quatro vezes o programa de temperatura (rampa, } \\
\text { tempo de aquecimento) para condicionamento do carbeto } \\
\text { de zircônio em elevada temperatura a : } 150{ }^{\circ} \mathrm{C}(1,10 \mathrm{~s}) \text {, } \\
600{ }^{\circ} \mathrm{C}(10,15 \mathrm{~s}), 1100{ }^{\circ} \mathrm{C}(10,5 \mathrm{~s}), 1400{ }^{\circ} \mathrm{C}(10,10 \mathrm{~s}), \\
1500{ }^{\circ} \mathrm{C}(3,5 \mathrm{~s}), 1600{ }^{\circ} \mathrm{C}(1,1 \mathrm{~s}), 1700{ }^{\circ} \mathrm{C}(1,1 \mathrm{~s}), \\
1800{ }^{\circ} \mathrm{C}(1,1 \mathrm{~s}), 1900{ }^{\circ} \mathrm{C}(1,1 \mathrm{~s}), 2000{ }^{\circ} \mathrm{C}(1,1 \mathrm{~s})\end{array}$ \\
\hline
\end{tabular}

a Todos as etapas do programa foram efetuadas com uma vazão de $250 \mathrm{~mL} \mathrm{~min}^{-1}$ de Ar.

Tabela 3. Programa de temperatura para estudo das curvas de temperatura de pirólise e atomização para $\mathrm{Au}, \mathrm{Ag}, \mathrm{Bi}, \mathrm{Cd}, \mathrm{Pb}$ e $\mathrm{Sn}$ em meio contendo ácido nítrico $0,2 \% \mathrm{v} / \mathrm{v}$ e em etanol

\begin{tabular}{ccccc}
\hline Etapa & Temperatura/ ${ }^{\circ} \mathrm{C}$ & Rampa/s & Patamar/s & $\begin{array}{c}\text { Vazão de Ar } \\
\mathrm{mL} \mathrm{min}{ }^{-1}\end{array}$ \\
\hline 1 & 90 & 5 & 10 & 250 \\
2 & $140\left(\mathrm{HNO}_{3}\right)$, & 5 & 10 & 250 \\
& $120($ etanol) & & & \\
3 & Variável & 10 & 20 & 250 \\
$4 *$ & Variável & 0 & 5 & 0 \\
5 & 2600 & 1 & 5 & 250 \\
6 & 20 & 1 & 5 & 250 \\
\hline
\end{tabular}

* leitura nesta etapa
Tabela 4. Programa de temperatura para estudo do efeito de diferentes concentrações de $\mathrm{Zr}$ sobre os sinais de $\mathrm{Bi}$ e $\mathrm{Cd}$ em meio contendo ácido nítrico $0,2 \% \mathrm{v} / \mathrm{v}$

\begin{tabular}{|c|c|}
\hline Passo & Ações e parâmetros \\
\hline 1 & $\begin{array}{l}\text { Pipetar } 2 \mu \mathrm{L} \text { de solução de } \mathrm{Zr}\left(500 \mu \mathrm{g} \mathrm{L}^{-1}\right) \text { sobre a } \\
\text { plataforma }\end{array}$ \\
\hline 2 & $\begin{array}{l}\text { Aplicar o seguinte programa de aquecimento } \text { (rampa, }^{\text {a }} \text { tempo de permanência) para secagem e pirólise e limpeza } \\
\text { do modificador: } 90{ }^{\circ} \mathrm{C}(5,15 \mathrm{~s}), 140{ }^{\circ} \mathrm{C}(5,15 \mathrm{~s}), 1000^{\circ} \mathrm{C} \\
(10,10 \mathrm{~s}), 2000{ }^{\circ} \mathrm{C}(0,5 \mathrm{~s}), 20^{\circ} \mathrm{C}(1,5 \mathrm{~s})\end{array}$ \\
\hline 3 & Pipetar $20 \mu \mathrm{L}$ de solução do analito \\
\hline 4 & $\begin{array}{l}\text { Aplicar o programa de temperatura (rampa, tempo de } \\
\text { aquecimento) para leitura do sinal analítico: } 90{ }^{\circ} \mathrm{C}(5 \text {, } \\
10 \mathrm{~s}), 140{ }^{\circ} \mathrm{C}(5,20 \mathrm{~s}), 800{ }^{\circ} \mathrm{C}-\mathrm{Bi} ; 500{ }^{\circ} \mathrm{C}-\mathrm{Cd} \text { e } 1200{ }^{\circ} \mathrm{C} \\
-\mathrm{Sn}(10,5 \mathrm{~s}), 1300{ }^{\circ} \mathrm{C}-\mathrm{Bi} \text { e } \mathrm{Cd} \text { e } 1700{ }^{\circ} \mathrm{C}-\mathrm{Sn}(10 \text {, } \\
10 \mathrm{~s}), 2200{ }^{\circ} \mathrm{C}(1,5 \mathrm{~s}), 20(1,5 \mathrm{~s})\end{array}$ \\
\hline
\end{tabular}

a Todos as etapas do programa foram efetuadas com uma vazão de $250 \mathrm{~mL} \mathrm{~min}^{-1}$ de Ar, exceto o passo de leitura que foi com parada do fluxo de gás.

\section{Procedimento de pré-concentração}

Para se testar em uma matriz real a eficiência analítica do Zr permanente, optou-se por aplicar aos elementos que obtiveram elevada sensibilidade em meio etanol, caso do bismuto, ouro e prata, pré-concentração a partir de digeridos de ácidos certificados eluídos com o etanol. O sistema de pré-concentração em fluxo e os procedimentos básicos utilizados para a pré-concentração foram descritos em detalhes anteriormente ${ }^{49}$. Para o sistema em fluxo, foi empregada uma bomba persiltáltica da Ismatec (Glattbrugg-Zürich, No.73315-15) equipada com tubos de tygon de vários diâmetros para solução aquosa e tubos "solvent-flex" para o etanol. Um injetor-comutador manual e uma mini-coluna preenchida com silica $\mathrm{C}_{18}$ comercial da Perkin Elmer (No. B050-4047), foram empregados. Em uma primeira etapa, com o injetor-comutador na posição de carga, a coluna é carregada com $10 \mathrm{~mL}$ de soluções de calibração ou amostras contendo 0,1\% de DDTP e $1 \mathrm{~mol} \mathrm{~L}^{-1} \mathrm{em}$ ácido clorídrico. As otimizações de complexação (concentração de DDTP e de HCL) foram obtidas anteriormente ${ }^{49}$. Na próxima etapa, o injetor foi comutado manualmente à sua segunda posição, permitindo que $500 \mu \mathrm{L}$ de etanol passe pela mini-coluna em sentido reverso da carga, eluindo assim o material adsorvido na minicoluna. O eluato foi coletado nos copos do amostrador automático do espectrômetro e imediatamente medida a concentração do analito para evitar volatilização do etanol e alterações na solução. Finalmente, a coluna foi lavada com $2 \mathrm{~mL}$ de etanol, mantendo o injetor na segunda posição, e com $5 \mathrm{~mL}$ de água depois de devolver o injetor à primeira posição. Este passo de limpeza foi eficiente para Bi e Au, mas não para Ag, para o qual a primeira limpeza foi com $3 \mathrm{~mL}$ de etanol (em sentido reverso da carga) e a segunda com $5 \mathrm{~mL}$ de ácido nítrico $1,0 \mathrm{~mol} \mathrm{~L}^{-1}$ (no sentido da carga). Todas as soluções foram substituídas manualmente junto à bomba peristáltica, intercaladas por um volume mínimo de ar que entra na tubulação. Foram otimizados fluxos de $1,2 \mathrm{~min}^{-1} \mathrm{de}$ $\mathrm{mL}$ e $0,36 \mathrm{~min}^{-1} \mathrm{de} \mathrm{mL}$, respectivamente para a primeira e segunda etapas de limpeza.

\section{RESULTADOS E DISCUSSÃO}

Para efeito de comparação do comportamento térmico de cada elemento investigado submetido ao programa de aquecimento (Tabela 3), em cada uma das superfícies, foram obtidas curvas de temperatura 
de pirólise e de atomização para cada analito em cada uma das situações, ou seja, em meio ácido nítrico $0,2 \%$ v/v e em meio etanol, atomizados diretamente a partir da plataforma de L'vov sem uso de modificador, empregando a mistura de $\mathrm{Pd}$ e $\mathrm{Mg}$ e um recobrimento com $500 \mu \mathrm{g}$ de $\mathrm{Zr}$ sobre a plataforma.

As medidas dos sinais em meio contendo ácido nítrico foram feitas em triplicatas, com desvios inferiores a 7\%. As medidas em etanol foram feitas em duplicata, exceto quando o desvio entre estas se aproximasse de $10 \%$, quando então mais uma ou duas medidas eram feitas até o desvio se tornar aceitável (abaixo de 10\%).

O procedimento empregado para a obtenção da plataforma recoberta com o $\mathrm{Zr}$ foi desenvolvido a partir dos estudos feitos anteriormente para obtenção de cobertura da plataforma com metais nobres, Ir e $\mathrm{Rh}^{18} \mathrm{e} \mathrm{Ru}^{19}$, combinado com estudos feitos com tratamento de tubo ou plataforma com elementos formadores de carbetos $(\mathrm{Zr}$ e W) ${ }^{6,34,35}$. Nestes últimos trabalhos ${ }^{34,35}$ após a obtenção de uma camada recoberta com $\mathrm{W}$, foi aplicado um programa de condicionamento térmico efetuado em dois passos para assegurar a transformação dos óxidos de tungstênio e/ou oxi-carbetos de tungstênio em carbetos do metal. Considerando o comportamento térmico relativamente similar entre $\mathrm{W}$ e $\mathrm{Zr}^{9,59}$, estes passos foram aqui adotados (etapas 4 e 5 da Tabela 2), com semelhante propósito.

\section{Prata}

Na Figura 1 estão representadas as curvas obtidas para Ag. Nas discussões empregar-se-ão a sigla tp referente à temperatura de pirólise, ta para a de atomização, e mo para massa característica (mo é a massa do analito em pg que gera um sinal de absorvância integrada de $0,0044 \mathrm{~s}$ ).

As curvas de temperatura de pirólise para $\mathrm{Ag}$ sem modificador nos dois meios químicos foram obtidas empregando ta de $1800{ }^{\circ} \mathrm{C}$

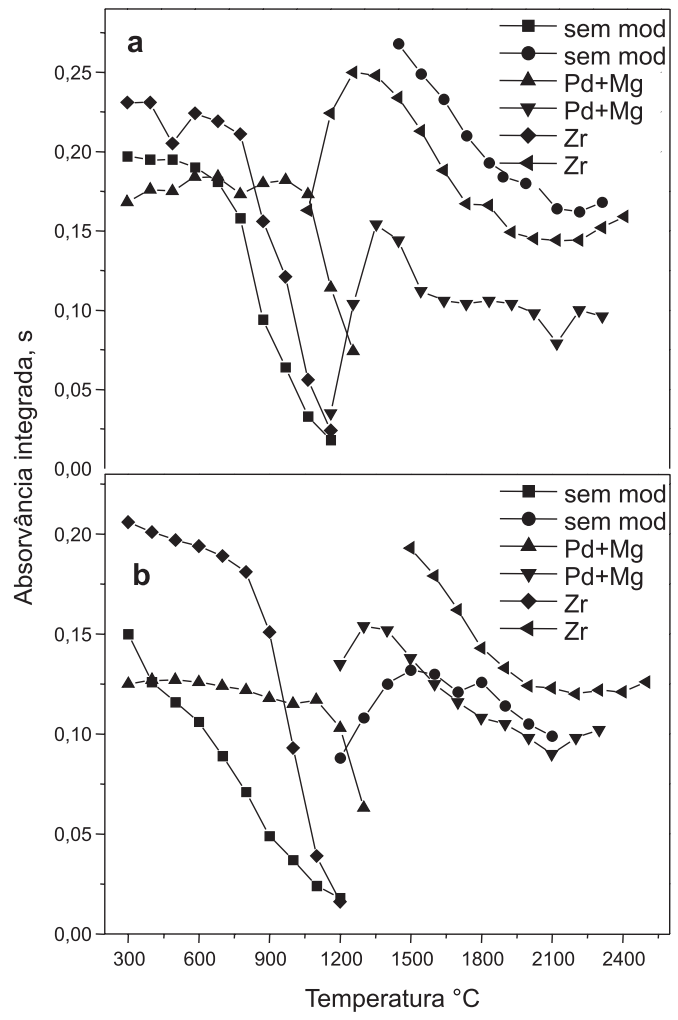

Figura 1. Curvas de temperatura de pirólise e de atomização para 70 pg de Ag, solubilizados em ácido nítrico $0,2 \% \mathrm{v} / \mathrm{v}($ a) e em etanol (b), para três diferentes coberturas da plataforma (recomendada pelo fabricante para uso com soluções em meio ácido nítrico $0,2 \% \mathrm{v} / \mathrm{v}$ e com Pd e Mg como modificadores ${ }^{7}$ ). É interessante observar-se a baixa sensibilidade observada para este metal quando solubilizado em meio etanol. Sem uso de modificador, o sinal de absorvância é baixo mesmo em temperatura de $300{ }^{\circ} \mathrm{C}$ e diminui ainda mais com o aumento da tp. Por outro lado, com uso de Pd e Mg a Ag em etanol parece estabilizada até cerca de $1000{ }^{\circ} \mathrm{C}$, permanecendo, contudo, em todo patamar uma baixa sensibilidade (mo de 2,0 pg). Apenas com uso de superfície recoberta com $\mathrm{Zr}$ em meio etanol o metal tem sensibilidade mais elevada, pelo menos até ca. de $800{ }^{\circ} \mathrm{C}$ (mo de 1,5 pg). Deve-se ressaltar que com a superfície de $\mathrm{Zr}$ uma ta de $1500{ }^{\circ} \mathrm{C}$ foi empregada para a obtenção da curva de temperatura de pirólise. Em meio ácido nítrico a sensibilidade é mais elevada para o metal mesmo sem modificador, no qual é estável até cerca de $600{ }^{\circ} \mathrm{C}$ (mo de 1,5 pg). Com uso de Pd e Mg em meio ácido nítrico, a estabilização ocorre até $1100{ }^{\circ} \mathrm{C}$ (mo de $1,6 \mathrm{pg}$ ). Com a camada de $\mathrm{Zr}$, neste meio, a sensibilidade é maior, pelo menos até $800^{\circ} \mathrm{C}$ (mo de $1,2 \mathrm{pg}$ ). Em relação às ta, de forma geral, observa-se uma tendência para que a maior sensibilidade esteja entre as temperaturas de $1300-1500{ }^{\circ} \mathrm{C}$, o que difere da ta recomendada de $1800{ }^{\circ} \mathrm{C}$. Em meio contendo ácido nítrico, observa-se boa sensibilidade sem modificador ou com $\mathrm{Zr}$ com ta em 1500 e $1300-1400{ }^{\circ} \mathrm{C}$, respectivamente. Em etanol, empregando uma ta de $1500{ }^{\circ} \mathrm{C}$ a mo observada foi de $1,5 \mathrm{pg}$.

\section{Ouro}

Na Figura 2 estão apresentadas as curvas obtidas para Au. A curva de temperatura de pirólise, na ausência de modificador em meio contendo ácido nítrico, foi obtida empregando uma ta de $1800{ }^{\circ} \mathrm{C}$. Uma primeira observação importante em relação à Figura 2 é que sem uso de modificador químico, a sensibilidade em meio contendo ácido nítrico é praticamente a metade daquela observada

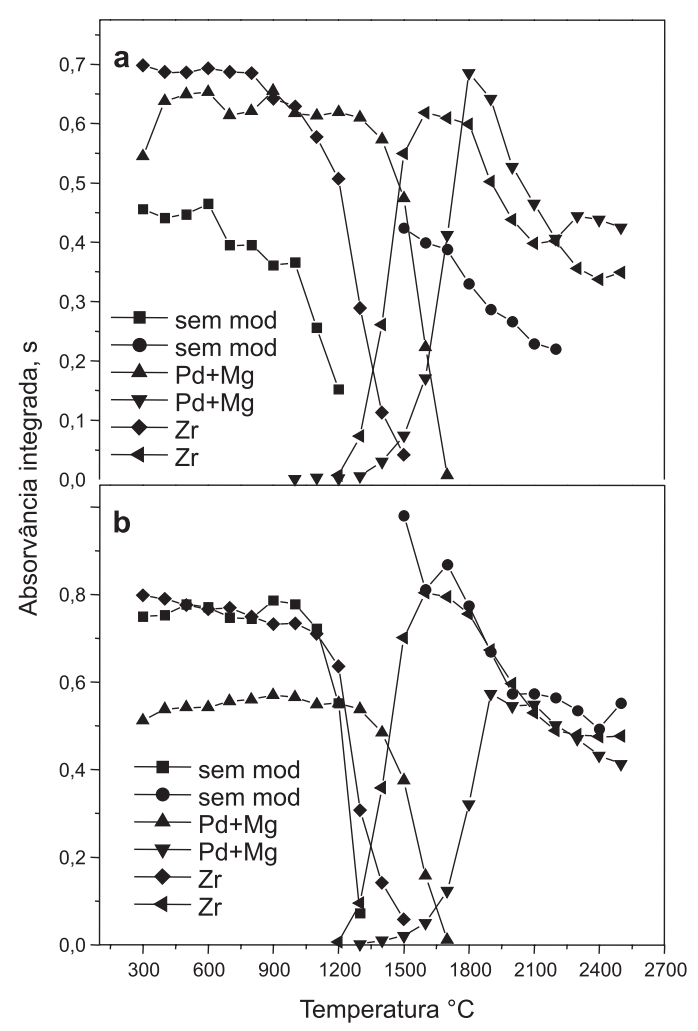

Figura 2. Curvas de temperatura de pirólise e de atomização para $1 \mathrm{ng}$ de $A u$, solubilizados em ácido nítrico $0,2 \% \mathrm{v} / v(a)$ e em etanol (b) 
em etanol. Neste último meio, com uma tp de $1000-1100{ }^{\circ} \mathrm{C}$ a mo obtida (cerca de $5,7 \mathrm{pg}$ ) é quase a metade daquela apontada pelo fabricante do equipamento empregado ${ }^{7}$ para este metal em meio ácido nítrico 0,2\% v/v e com Pd e Mg como modificadores químicos. A baixa sensibilidade observada para Au em meio de ácido nítrico pode ser devida à menor solubilidade do metal neste meio. Em estudo sobre comportamento de diferentes modificadores aplicados em solução para uma série de analitos, incluído o Au, Tsalev e Slaveykova ${ }^{8}$ estudaram a maioria dos elementos solubilizados em ácido nítrico $0,2 \% \mathrm{v} / \mathrm{v}$, com exceção de alguns, como o Au que foi solubizado em meio $0,2 \% \mathrm{v} / \mathrm{v}$ de ácido clorídrico. Com o emprego da mistura de Pd e $\mathrm{Mg}$, por outro lado, obteve-se uma mo de cerca de 8,0 pg em meio contendo ácido nítrico com estabilização térmica do analito até cerca de $1300{ }^{\circ} \mathrm{C}$, o mesmo ocorrendo em meio etanol, porém, com uma sensibilidade mais baixa. Empregando-se a cobertura de $\mathrm{Zr}$, de modo oposto, maior sensibilidade foi obtida com etanol comparativamente ao meio ácido nítrico. Observando-se as curvas de atomização pode-se concluir que com uma ta de $1500{ }^{\circ} \mathrm{C}$, em meio contendo etanol e sem uso de modificador a maior sensibilidade pode ser obtida (mo de 5,0 pg). Com uso de $\mathrm{Pd}$ e $\mathrm{Mg}$ as ta nas quais ocorre maior sensibilidade estão entre $1800-1900{ }^{\circ} \mathrm{C}$, enquanto que com a cobertura de $\mathrm{Zr}$, valores de ta entre $1500-1700{ }^{\circ} \mathrm{C}$ parecem possibilitar maior sensibilidade para este metal. Com a plataforma assim recoberta, mo de 5,5 e 8,0 pg foram obtidas, respectivamente, para o metal solubilizado em etanol e em ácido nítrico.

\section{Bismuto}

As curvas de temperatura de pirólise e de atomização obtidas para Bi em cada um dos meios químicos e modificadores estão representadas na Figura 3. A observação mais importante que pode ser obtida pela análise da Figura 3 diz respeito à elevada sensibilidade observada para este metal quando solubilizado em etanol e empre-

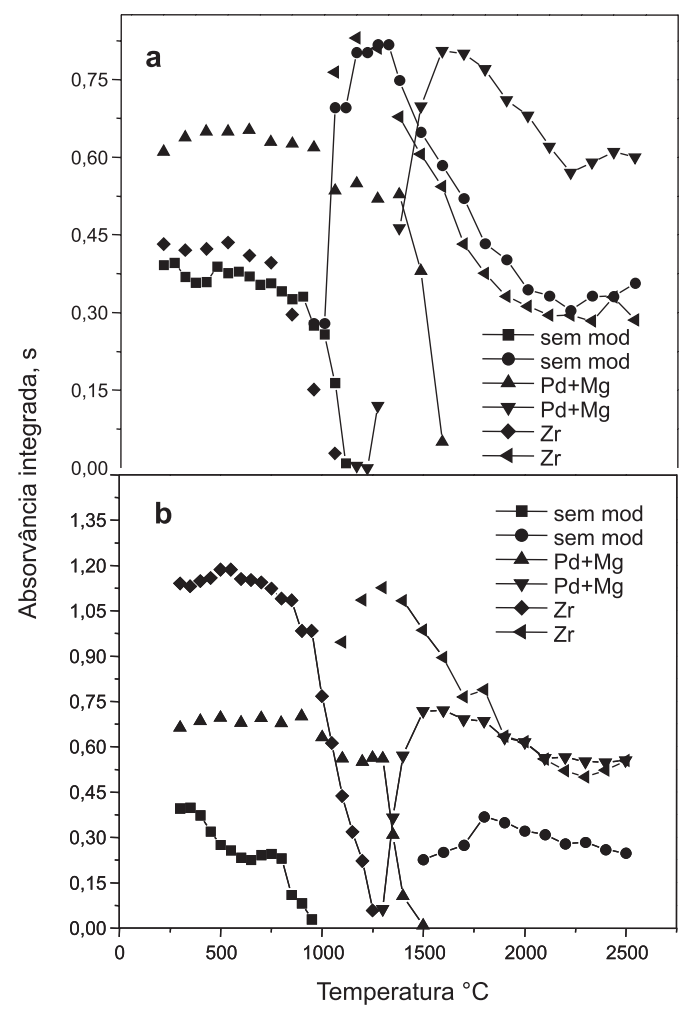

Figura 3. Curvas de temperatura de pirólise e de atomização para 2 ng de $\mathrm{Bi}$, solubilizados em ácido nítrico $0,2 \% \mathrm{v} / \mathrm{v}(\mathrm{a})$ e em etanol (b) gando-se a camada de $\mathrm{Zr}$ como modificador químico. Com uma estabilidade térmica até cerca de $800^{\circ} \mathrm{C}$ e com uma ta entre $1300-1400{ }^{\circ} \mathrm{C}$, uma mo de cerca de 4,0 pg foi obtida. Considerando-se que o valor para mo sugerido pelo fabricante para este metal em ácido nítrico 0,2\% v/v e com a mistura de Pd e Mg como modificador é de 19,0 pg, este valor aqui obtido de 4,0 pg pode ser importante e sugere a possibilidade de obtenção de um limite de detecção mais baixo para este analito, principalmente considerando-se que com este procedimento a cobertura de $\mathrm{Zr}$ é limpa durante o processo de deposição deste modificador sobre a plataforma. Como este metal na maioria das matrizes investigadas por ET AAS apresenta-se em concentrações baixas (freqüentemente mais baixa que o LD da técnica), uma solubilização deste em etanol e uso da plataforma recoberta com $\mathrm{Zr}$ pode se tornar uma alternativa, o que será investigado em etapa posterior deste estudo. Em meio contendo ácido nítrico, por outro lado, as mo obtidas (cerca de 12,0 pg) com esta mesma cobertura, são cerca de três vezes mais elevadas do que em meio etanol. Com a mistura de $\mathrm{Pd}$ e $\mathrm{Mg}$, valores intermediários foram obtidos de forma similar para o metal em etanol e em ácido nítrico. Em relação às temperaturas de atomização, sem modificador ou com uso da camada de $\mathrm{Zr}$, em valores de ta de cerca de $1300{ }^{\circ} \mathrm{C}$, máxima sensibilidade foi obtida para o analito, diferente do que ocorre com uso da mistura de $\mathrm{Pd}$ e $\mathrm{Mg}$, para a qual em valores de ta entre $1700-1800{ }^{\circ} \mathrm{C}$ uma sensibilidade maior foi observada.

\section{Cádmio}

Os resultados obtidos para o $\mathrm{Cd}$ em ácido nítrico e etanol com as três superfícies modificadoras investigadas neste estudo estão apresentados na Figura 4. Em relação à curva de temperatura de pirólise, sem uso de modificador (foi empregada uma ta de $1650{ }^{\circ} \mathrm{C}$ ), em meio ácido a sensibilidade é baixa e o metal estável até cerca de $500{ }^{\circ} \mathrm{C}$, enquanto que em etanol, já a partir de $300-350{ }^{\circ} \mathrm{C}$ ocorre

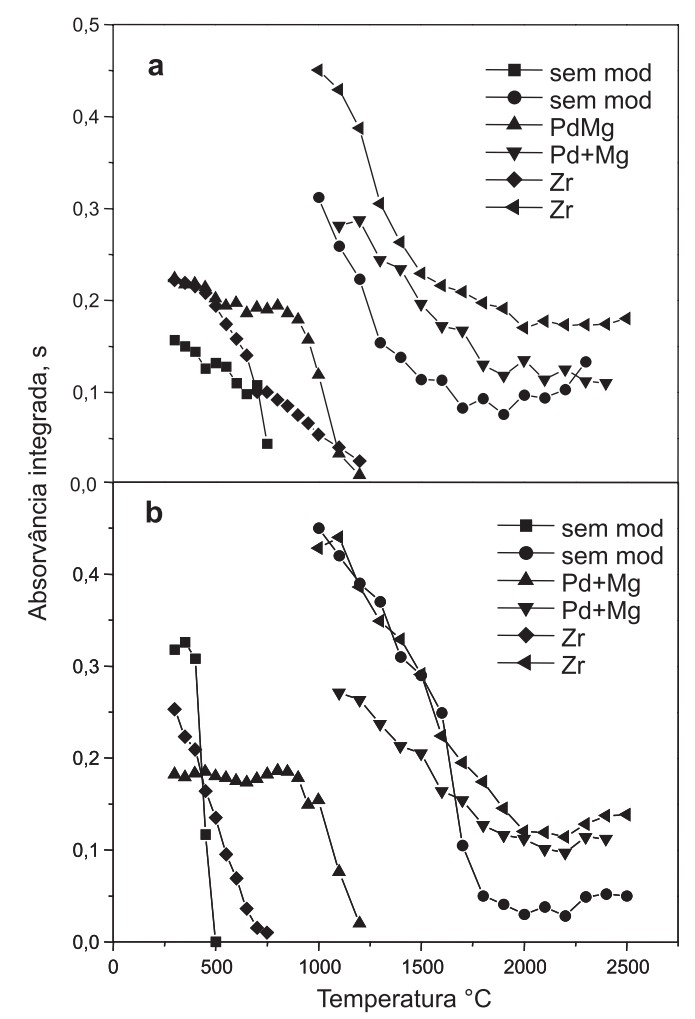

Figura 4. Curvas de temperatura de pirólise e de atomização para 40 pg de $C d$, solubilizados em ácido nítrico $0,2 \%$ v/v (a) e em etanol (b) 
uma queda brusca no sinal. Com uso de Pd e Mg, por outro lado, tanto em meio ácido como em etanol o metal é estável até cerca de $800{ }^{\circ} \mathrm{C}$ (mo de $0,9 \mathrm{pg}$ ). Com $\mathrm{Zr}$, com esta ta, os sinais para $\mathrm{Cd}$ nos dois meios são baixos e pouco estáveis à medida que se aumenta a tp. Em relação às curvas de temperaturas de atomização observadas, de forma geral, para todas as superfícies modificadoras e nos dois meios parece ocorrer uma tendência de diminuição na sensibilidade, aumentando-se a ta. Observe-se que o fabricante recomenda o uso de ta de $1650{ }^{\circ} \mathrm{C}$ para o $\mathrm{Cd}$ com $\mathrm{Pd}$ e $\mathrm{Mg}$ como modificadores químicos (mo de $0,5 \mathrm{pg}$ ). No presente estudo, as maiores sensibilidades foram observadas para o Cd em meio etanol, tanto sem modificador, com o uso de camada recoberta com $\mathrm{Zr}$ (mo de 0,4 pg) e também com $\mathrm{Pd}$ e $\mathrm{Mg}$ em ácido nítrico. Contudo, com este último com as ta de $1600-1700{ }^{\circ} \mathrm{C}$, a mo obtida foi de $1,0 \mathrm{pg}$, diminuindo para $0,6 \mathrm{pg}$ com ta entre $1100-1200{ }^{\circ} \mathrm{C}$.

\section{Chumbo}

Os resultados obtidos nos dois meios estudados para $\mathrm{Pb}$ com as três superfícies estão apresentados na Figura 5. A ta inical empregada para o $\mathrm{Pb}$ nos dois meios químicos foi de $1800{ }^{\circ} \mathrm{C}$ (recomendada) $)^{7}$. Sem uso de modificador, o sinal é estabilizado até cerca de $600-700{ }^{\circ} \mathrm{C}$ (mo de 15 e $24 \mathrm{pg}$, respectivamente, para meio ácido e etanol). Empregando $\mathrm{Pd}$ e $\mathrm{Mg}$, até $1000^{\circ} \mathrm{C}$ pode-se observar estabilidade para o metal com mo de 14 e $19 \mathrm{pg}$, respectivamente, para os meios ácido e etanol. Com a camada de $\mathrm{Zr}$, por outro lado, a estabilidade térmica do $\mathrm{Pb}$ é menor (até ca de $700-800{ }^{\circ} \mathrm{C}$ ) com maior sensibilidade com mo de ca. de $11,5 \mathrm{pg}$. Observe-se contudo que para a camada de $\mathrm{Zr}$, uma ta de $1400{ }^{\circ} \mathrm{C}$ foi empregada para obtenção da curva de tp. Como tem-se, de forma geral, observado que para modificadores permanentes uma ta mais baixa gera melhor mais sensibilidade do que os valores normalmente recomendados para uso com Pd e Mg, fez-se um estudo com tp em $300{ }^{\circ} \mathrm{C}$ e variando-se a ta na faixa crítica de 1300 a $2000{ }^{\circ} \mathrm{C}$. A sensibilidade foi nitidamente maior em cerca de $1300-1400{ }^{\circ} \mathrm{C}$, motivo pelo qual optou-se por este valor para o estudo do $\mathrm{Pb}$ com o $\mathrm{Zr}$ como modificador. Através das curvas de temperaturas de atomização, pode-se observar que, empregando-se $\mathrm{Pd}$ e $\mathrm{Mg}$ em meio contendo ácido nítrico, os valores mais elevados foram obtidos entre $1600-1700{ }^{\circ} \mathrm{C}$ (mo de $14 \mathrm{pg}$ ), sendo estes mais baixos em meio etanol. $\mathrm{O}$ uso de temperaturas de atomização mais baixas (cerca de $1300{ }^{\circ} \mathrm{C}$ ) parece adequado, sendo mais sensível para o metal em meio ácido sem modificador ou quando do uso de $\mathrm{Zr}$ nos dois meios químicos. Nestes casos foram obtidas massas características de cerca de 11,$5 ; 11$ e 10 pg, respectivamente, para meio ácido sem modificador, meio ácido com $\mathrm{Zr}$ e etanol com Zr.

\section{Estanho}

Os resultados observados para o estanho estão representados pela Figura 6. Um primeiro dado interessante que pode ser observado na Figura 6 diz respeito à baixa sensibilidade para $\mathrm{Sn}$ solubilizado em etanol quando não é empregado modificador, comparativamente àquela obtida para o elemento em meio ácido (a qual é cerca de três vezes mais elevada que no meio etanóico). A curva de temperatura de pirólise para o metal sem uso de modificador nos dois meios foi a recomendada ${ }^{7}$ de $2300{ }^{\circ} \mathrm{C}$. Com emprego da mistura Pd e $\mathrm{Mg}$ (com esta mesma ta), uma temperatura de cerca de $1600{ }^{\circ} \mathrm{C}$ pode ser empregada para o $\mathrm{Sn}$ em ambos os meio químicos (com uma sensibilidade levemente menor em meio etanol, mo de 11,8 e 12,8 pg, respectivamente, para meio ácido e etanol). A mesma consideração pode ser feita para a ta observada com uso de Pd e Mg nos dois meios, para os quais em valores acima de $2000{ }^{\circ} \mathrm{C}$, o metal parece estabilizado. Com uso de camada de $\mathrm{Zr}$ como modificador químico $\left(2300{ }^{\circ} \mathrm{C}\right.$

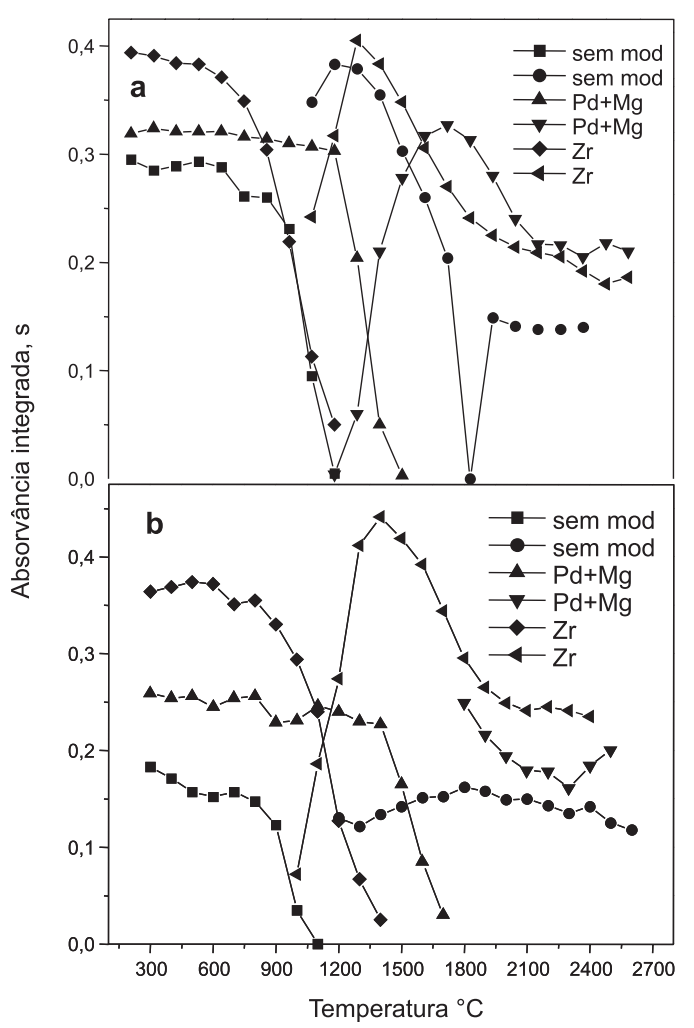

Figura 5. Curvas de temperatura de pirólise e de atomização para $1 \mathrm{ng}$ de $\mathrm{Pb}$, solubilizados em ácido nítrico $0,2 \% \mathrm{v} / \mathrm{v}($ a) e em etanol (b)

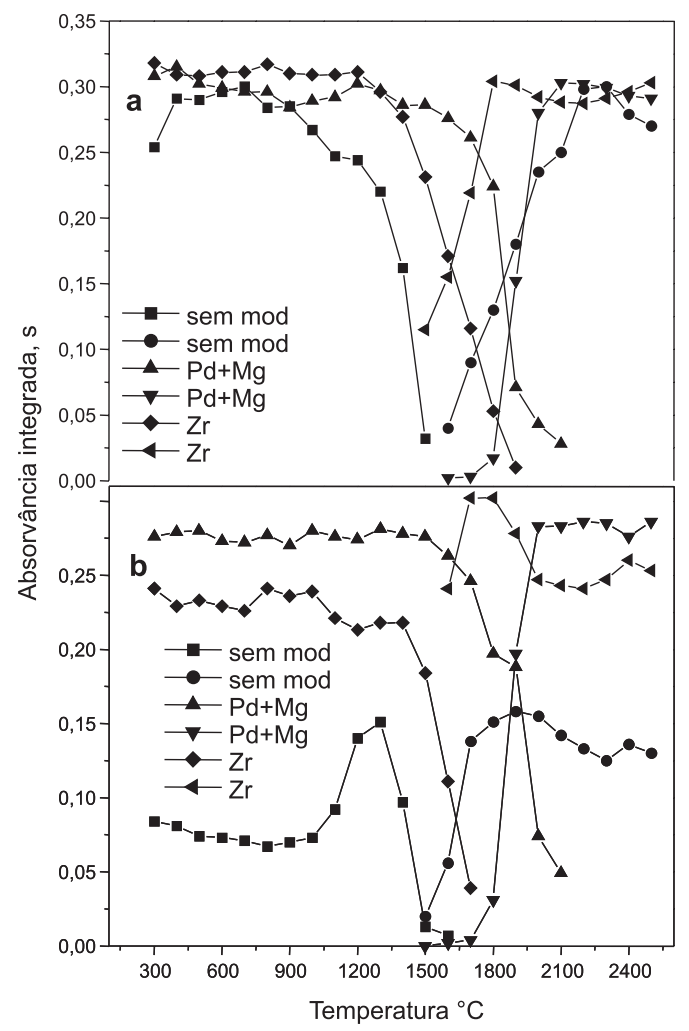

Figura 6. Curvas de temperatura de pirólise e de atomização para 1 ng de Sn, solubilizados em ácido nítrico $0,2 \% \mathrm{v} / \mathrm{v}($ a) e em etanol (b) 
para ta), uma mo de $11,3 \mathrm{pg}$ pode ser obtida com $1300{ }^{\circ} \mathrm{C}$ de tp em meio ácido nítrico. Com este modificador, em etanol, a melhor condição obtida foi para tp de $1200{ }^{\circ} \mathrm{C}$ e ta de $2000{ }^{\circ} \mathrm{C}$ com mo de cerca de 14,0 pg. Apesar de se observar um sinal mais elevado (mo de $11,7 \mathrm{pg}$ ) com ta de $1700{ }^{\circ} \mathrm{C}$, o sinal transiente nesta temperatura era mais irregular do que aquele, simétrico e bem formado, observado com ta acima de $2000{ }^{\circ} \mathrm{C}$.

\section{Influência da massa de zircônio sobre o sinal de absorvância de Bi}

Para se averiguar a influência da massa de zircônio aplicada sobre a plataforma no sinal analítico de bismuto e de ouro, foram efetuados estudos com crescentes massas do $\mathrm{Zr}$, para uma solução de 3 ng de Bi em meio contendo ácido nítrico. O programa de aquecimento empregado para a obtenção dos sinais observados está apresentado na Tabela 4. Neste programa, inicialmente uma alíquota da solução do modificador é introduzida no forno de grafite, sendo submetida às etapas de secagem e pirólise para a obtenção do metal na forma reduzida sobre a plataforma, subseqüentemente o amostrador automático coleta e introduz no forno uma alíquota da solução analítica do metal investigado, aplicando as etapas finais do programa, com a secagem, pirólise, atomização (com a leitura do sinal do analito) e limpeza.

É importante observar que a temperatura máxima empregada no programa de temperatura de forno (etapa de limpeza) foi de $2200{ }^{\circ} \mathrm{C}$. É de se supor que nesta temperatura não ocorram perdas do $\mathrm{Zr}$ depositado sobre a plataforma, já que o ponto de ebulição do metal é elevado. Este fato é importante já que se consideram valores aditivos para as massas depositadas na plataforma.

De acordo com Lima et $a l . .^{34}$, empregando uma plataforma recoberta com $\mathrm{W}$ como modificador para Se, com o uso de temperaturas mais elevadas do que $2100-2200{ }^{\circ} \mathrm{C}$, o número de ciclos de atomização diminuiu de 300-350 para menos de 200, o que foi atribuído a perdas por volatilização do $\mathrm{W}$ nas temperaturas mais elevadas. No presente estudo, mesmo que ocorram algumas perdas de $\mathrm{Zr}$, estas devem ser pequenas, podendo-se considerar que os valores reais de massa de $\mathrm{Zr}$ depositados sobre a plataforma são aproximados daqueles indicados. Em um primeiro momento, foram registrados os brancos obtidos com o programa da Tabela 4, a partir de soluções de ácido nítrico $0,2 \%$ v/v (sem o analito), sendo estes valores observados descontados nos estudos feitos com a solução do analito. As temperaturas de pirólise e atomização, bem como as demais etapas do programa de forno, foram otimizadas no estudo anterior.

Neste estudo, observou-se um aumento no sinal do analito com aumento da massa de zircônio até cerca de $25 \mathrm{mg}$, valor a partir do qual torna-se praticamente constante com o aumento da massa do modificador.

Durante os estudos observou-se que, pelo menos por 500 ciclos de atomização, o sinal de absorvância permanece constante para ouro em meio ácido nítrico (elemento para o qual as temperaturas de pirólise e atomização são mais elevadas), comprovando a eficácia do $\mathrm{Zr}$ como modificador permanente.

\section{Figuras de mérito}

A Tabela 5 apresenta as massas características obtidas para os seis analitos investigados nos dois meios químicos. O programa de temperatura de forno (com a plataforma tratada com zircônio permanente) para a determinação dos metais após a pré-concentração está apresentado na Tabela 6, enquanto que as características analíticas do método de pré-concentração estão reportadas na Tabela 7.

$\mathrm{O}$ fator de enriquecimento obtido (calculado pela razão das inclinações de curvas submetidas e não submetidas ao procedimento de pré-concentração) pode ser considerado bom para todos os metais, com limites de deteção da ordem de $\mu \mathrm{g} \mathrm{L}^{-1}$. O limite de detecção, LD, foi definido como a relação entre três vezes o desvio padrão de 10 medidas do branco após a pré-concentração e a inclinação da curva de calibração, também após a pré-concentração. Os coeficientes de correlação foram sempre superiores a 0,99.

Conforme pode-se observar na Tabela 8 , os valores determinados concordam com os valores certificados para os três metais estudados. Para a construção da curva de calibração, todas as soluções foram submetidas ao mesmo procedimento de pré-concentração que, posteriormente, o foram as amostras. Os desvios padrão relativos

Tabela 5. Massas características recomendadas e obtidas com diferentes modificadores químicos

\begin{tabular}{|c|c|c|c|c|c|c|c|}
\hline \multirow[t]{2}{*}{ Condição } & & \multirow[b]{2}{*}{$\mathrm{Ag}$} & \multicolumn{5}{|c|}{ Analito (pg) } \\
\hline & & & $\mathrm{Au}$ & $\mathrm{Bi}$ & $\mathrm{Cd}$ & $\mathrm{Pb}$ & $\mathrm{Sn}$ \\
\hline \multirow{3}{*}{$\begin{array}{l}\text { Recomendado } \\
\text { Sem modif. }\end{array}$} & $\mathrm{HNO}_{3}$ & 1,5 & 8,5 & 19,0 & 0,5 & 10,0 & 10,0 \\
\hline & $\mathrm{HNO}_{3}$ & 2,4 & 11,7 & 21,0 & 1,2 & 12,0 & 12,4 \\
\hline & Etanol & 1,4 & 5,9 & 19,5 & 0,6 & 13,6 & 26,4 \\
\hline \multirow[t]{2}{*}{$\mathrm{Pd}+\mathrm{Mg}$} & $\mathrm{HNO}_{3}$ & 1,7 & 8,1 & 14,0 & 0,8 & 13,2 & 14,4 \\
\hline & Etanol & 1,9 & 7,5 & 11,7 & 1,0 & 17,7 & 12,6 \\
\hline \multirow[t]{2}{*}{$\mathrm{Zr}(500 \mu \mathrm{g})$} & $\mathrm{HNO}_{3}$ & 1,6 & 7,0 & 12,7 & 1,3 & 10,1 & 14,2 \\
\hline & Etanol & 0,8 & 5,8 & 8,0 & 0,3 & 10,3 & 13,0 \\
\hline
\end{tabular}

Tabela 6. Programa de temperatura de forno para análise de $\mathrm{Ag}, \mathrm{Au}$ e Bi extraídos em etanol após pré-concentração em sistema de fluxo

\begin{tabular}{ccccc}
\hline Etapa & Temperatura/ ${ }^{\circ} \mathrm{C}$ & Rampa/s & $\begin{array}{c}\text { Tempo de } \\
\text { permanência/s }\end{array}$ & $\begin{array}{c}\text { Fluxo de Ar, } \\
\mathrm{mL} \mathrm{min}^{-1}\end{array}$ \\
\hline 1 & 90 & 5 & 10 & 250 \\
2 & 200 & 5 & 10 & 250 \\
3 & $600(\mathrm{Ag}, \mathrm{Bi})$, & 10 & 20 & 250 \\
& $900(\mathrm{Au})$ & & & \\
$4^{*}$ & $1300(\mathrm{Ag}, \mathrm{Bi})$, & 0 & 5 & 0 \\
5 & $1600(\mathrm{Au})$ & & & \\
5 & 2600 & 1 & 5 & 250 \\
6 & 20 & 1 & 5 & 250 \\
\hline
\end{tabular}

* leitura nesta etapa

Tabela 7. Características analíticas do sistema de pré-concentração empregando plataforma tratada com Zr permanente na determinação dos analitos

\begin{tabular}{ccccccc}
\hline Analito & $\begin{array}{c}\text { Inclinação* } \\
\mathrm{s}\left(\mu \mathrm{L} \mathrm{L}^{-1}\right)^{-1}\end{array}$ & $\begin{array}{c}\text { Inclinação** } \\
\mathrm{s}\left(\mu \mathrm{g} \mathrm{L}^{-1}\right)^{-1}\end{array}$ & $\begin{array}{c}\text { Fator de } \\
\text { enriquecimento }\end{array}$ & $\begin{array}{c}\text { Coeficiente } \\
\text { de correlação }\end{array}$ & $\begin{array}{c}\text { Faixa } \\
\mu \mathrm{L} \mathrm{L}^{-1}\end{array}$ & $\begin{array}{c}\mathrm{LD} \\
\mu \mathrm{L}^{-1}\end{array}$ \\
\hline $\mathrm{Ag}$ & 0.940 & 0.050 & 19.0 & 0.9933 & $01-0.5$ & 0.029 \\
$\mathrm{Au}$ & 0.760 & 0.016 & 14.5 & 0.9949 & $0.1-1.0$ \\
$\mathrm{Bi}$ & 0.600 & 0.015 & 15.5 & 0.9962 & $0.1-1.0$ & 0.020 \\
\hline
\end{tabular}

*após a pré-concentração, **em etanol, sem aplicação de pré-concentração. 
estão entre 3 a 18, \%, mostrando uma precisão razoável para um sistema de injeção de fluxo do tipo "home-made".

Table 8. Concentrações determinadas após pré-concentração em amostras certificadas de aços (NIST) em $\mathrm{mg} \mathrm{L}^{-1}, \mathrm{n}=3$

\begin{tabular}{cccc}
\hline Analito & Amostra & Obtido & Certificado* \\
\hline \multirow{2}{*}{$\mathrm{Ag}$} & SRM 361 & $3.8 \pm 0.7$ & 4.0 \\
& SRM 364 & $0.23 \pm 0.04$ & 0.2 \\
$\mathrm{Au}$ & SRM 361 & $0.38 \pm 0.01$ & $<0.5$ \\
& SRM 364 & $0.95 \pm 0.10$ & 1.0 \\
$\mathrm{Bi}$ & SRM 361 & $3.81 \pm 0.35$ & 4.0 \\
& SRM 364 & $8.37 \pm 0.43$ & 9.0 \\
\hline
\end{tabular}

* De acordo com o certificado o valor do desvio foi sempre inferior $\mathrm{a} \pm 1 \%$.

\section{CONCLUSÕES}

Com os metais solubilizados em etanol, a atomização direta sobre a plataforma sem uso de modificador mostrou-se apropriada apenas para $\mathrm{Au}$ e $\mathrm{Cd}$, especialmente quando empregando ta mais baixa que a recomendada. Para os outro metais, o uso de camada de $\mathrm{Zr}$ eleva a sensibilidade, de modo que para Ag obtém-se uma mo comparável àquela com uso de $\mathrm{Pd}$ e $\mathrm{Mg}$ em meio ácido. Para $\mathrm{Bi}$ e $\mathrm{Cd}$ o ganho em sensibilidade em etanol com $\mathrm{Zr}$ é pelo menos três vezes maior que os valores recomendados e normalmente citados para estes metais, sendo também maior a sensibilidade nesse meio (etanol e $\mathrm{Zr}$ ) para $\mathrm{Au}$. Em meio contendo ácido nítrico observa-se que para Ag boa sensibilidade pode ser obtida com $\mathrm{Zr}$ ou mesmo sem modificador, se for empregada uma ta mais baixa que a recomendada para uso com $\mathrm{Pd}$ e $\mathrm{Mg}$; para $\mathrm{Au}$ e $\mathrm{Sn}$ o uso de camada de $\mathrm{Zr}$ é comparável ao uso de Pd e Mg (com estabilidade térmica maior para $\mathrm{Au}$ com a mistura Pd e Mg). Para Bi, empregando-se uma ta mais baixa tanto sem modificador como com $\mathrm{Zr}$, a sensibilidade é duas a três vezes maior que o valor recomendado, o mesmo ocorrendo para $\mathrm{Cd}$ com $\mathrm{Zr}$ (em ta igualmente mais baixa). Para $\mathrm{Pb}$, empregando ta $\mathrm{e}$ tp mais baixas $\left(800 \mathrm{e} 1500{ }^{\circ} \mathrm{C}\right)$ que o recomendado, a mais alta sensibilidade pode ser obtida (o sinal médio de 0,3 observado com a mistura Pd e Mg, pode atingir 0,45 nas condições citadas com $\mathrm{Zr}$ ). A vida útil do tubo de grafite é de cerca de 500 ciclos, o que assegura o comportamento do $\mathrm{Zr}$ como modificador permanente.

Aplicando-se o uso do $\mathrm{Zr}$ permanente para receber o eluato (500 $\mu \mathrm{L}$ de etanol) de digeridos de ácidos certificados, após pré-concentração em fluxo em mini-coluna preenchida com sílica $\mathrm{C}_{18}$, os resultados concordaram com os certificados para bismuto, ouro e prata.

\section{AGRADECIMENTOS}

Ao CNPq (Conselho Nacional de Desenvolvimento Científico e Tecnológico) pela Bolsa de Produtividade em Pesquisa e à Pró-Reitoria de Pesquisa da UFMG pelo apoio financeiro.

\section{REFERÊNCIAS}

1. Slavin, W.; Manning, D. C.; Carnrick, G. R; At. Spectrosc. 1981, 2, 137.

2. Ediger, R. E.; At. Absorpt. Newsl. 1975, 14, 127.

3. Schlemmer, G.; Welz, B.; Spectrochim. Acta, Part B 1986, 41, 1157.

4. Welz, B.; Schlemmer, G.; Mudakavi, J. R.; J. Anal. At. Spectrom. 1988, 3, 93.

5. Welz, B.; Schlemmer, G.; Mudakavi, J. R.; J. Anal. At. Spectrom. 1992, 7, 1257.

6. Rademeyer, C.; Radziuk, B.; Romanova, N. ; Skaugset, N. P.; Skogstad, A.; Thomassen, Y.; J. Anal. At. Spectrom. 1995, 10, 739.
7. Bodenseewerk Perkin-Elmer, Überlingen, Analytical Techniques for Graphite furnace AAS, 1984. Part No. B010-0180 (B332/E).

8. Tsalev, D. L.; Slaveykova, V. I.; Spectrosc. Lett. 1992, 25, 221.

9. Tsalev, D.; D’Ulivo, A.; Lampugnani, A.; Di Marco, M.; Zamboni, R. J.; J. Anal. At. Spectrom. 1995, 10, 1003 .

10. Bulska, E.; Jedral , W.; J. Anal. At. Spectrom. 1995, 10, 49.

11. Bulska, E.; Kandler, W.; Hulanicki, A.; Spectrochim Acta, Part B 1996, $51,1263$.

12. Ni, Z-M.; Rao, Z.; Li, M.; Anal. Chim. Acta 1996, 334, 177.

13. Li, M.; Ni, Z-M.; Rao, Z.; Spectrochim. Acta, Part B 1998, 53, 1381.

14. Volynski, A. B.; Tikhomirov, S. V.; J. Anal. Chem. 1998, 53, 718.

15. Haldimann, M.; Venner, T. Y.; Zimmerli, B.; J. Trace Elements in Medicine and Biology 1996, 10, 31.

16. Cai, K.; McDonald, C. V.; Microchem. J. 1997, 57, 370.

17. Volinsky, A. B.; Krivan, W.; J. Anal. At. Spectrom. 1997, 12, 333.

18. Silva, J. B. B.; Giacomelli, M. B. O.; Souza, I. G.; Curtius, A. J.; Microchem. J. 1998, 60, 249.

19. Silva, J. B. B.; Silva, M. A. M.; Curtius, A. J.; Welz, B.; J. Anal. At. Spectrom. 1999, 14, 1737.

20. Silva, J. B. B.; Veiga, M. A. M.; Galindo, D.; Curtius, A. J.; Welz, B.; Talanta 2003, 60, 977.

21. Giacomelli, M. B. O.; Lima, M. C.; Stupp, V.; Silva, J. B. B.; Barrera, P. B.; Spectrochim Acta, Part B 2002, 57, 2151.

22. Magalhães, C. G.; Lelis, K. L. A.; Rocha, C. A.; Silva, J. B. B.; Anal. Chim. Acta 2002, 464, 323.

23. Nunes, B. R.; Magalhães, C. G.; Silva, J. B. B.; J. Anal. At. Spectrom. 2002, 17, 1335.

24. Linces, K. L. A.; Magalhães, C. G.; Rocha, C. A.; Silva, J. B. B.; Anal. Bioanal. Chem. 2002, 374, 1301.

25. Tsalev, D. L.; Slaveikova, V. I.; Mandjukov, P. B.; Spectrochim. Acta Rev. 1990, 13, 25

26 Fritzsche, H.; Wolfhard, W.; Knapp, G.; Ortner, H. M.; Talanta 1979, 26, 219.

27. Yan, X-P.; Ni, Z-M.; Spectrochim. Acta, Part B 1993, 48, 1315.

28. Ni, Z-M.; Zhang, D-Q.; Spectrochim. Acta, Part B 1995, 50, 1779.

29. Liu, Y-M.; Gong, B-L.; Xu, Y-L.; Li, Z-H.; Lin, T-Z.; Anal. Chim. Acta 1999, 292, 325.

30. Havezov, I.; Detcheva, A.; Rendl, J.; Mikrochim. Acta 1995, 119, 147.

31. Gong, B-L.; Liu, Y-M.; Xu, Y-L.; Li, Z-H.; Lin, T-Z.; Talanta 1995, 2, 1419.

32. Yang, W-M.; Ni, Z-M.; Spectrochim. Acta, Part B 1997, 52, 241.

33. Shoukry, A. F.; Issa, Y. M.; Farghaly, R. A.; Grasserbauer, M.; Puxbaum, H.; Rendl, J.; Fresenius J. Anal. Chem. 1998, 360, 650.

34. Lima, E. C.; Krug, F. J.; Jackson, K. W.; Spectrochim. Acta, Part B 1998, $53,1791$.

35. Lima, E. C.; Krug, F. J.; Ferreira, A. T.; Barbosa Jr., F.; J. Anal. At. Spectrom. 1999, 14, 269.

36. Welz, B.; Yin, X.; Sperling, M.; Anal. Chim. Acta 1992, 261, 477.

37. Welz, B.; Sperling, M.; Su, X.; Fresenius J. Anal. Chem. 1993, 46, 550.

38. Sperling, M.; Yin, X-F.; Welz, B.; Fresenius J. Anal. Chem. 1992, 343, 754

39. Ma, R. L.; Adams, F.; Anal. Chim. Acta 1995, 317, 215.

40. Ma, R. L.; Van Mol, W.; Adams, D. F.; At. Spectrosc. 1996, 17, 176

41. Yan, X-P.; Sperling, M.; Welz, B.; Anal Chem. 1999, 71, 4353.

42. Yan, X-P.; Van Mol, W.; Adams, F.; Analyst 1996, 121, 1061.

43. Sperling, M.; Yan, X-P.; Welz, B.; Spectrochim. Acta, Part B 1996, 51, 1891.

44. Yan, X-P.; Adams, F.; J. Anal. At. Spectrom. 1997, 12, 459.

45. Yvanova, E.; Yan, X-P.; Van Mol, W.; Adams, F.; Analyst 1997, 122, 667.

46. Soylak, M.; Elci, L.; Int. J. Environ. Anal. Chem. 1997, 66, 51

47. Silva, J. B. B.; Giacomelli, M. B. O.; Curtius, A. J.; Analyst 1999, 124, 1249

48. Hiraide, M.; Mikuni, Y.; Kawaguchi, H.; Analyst 1994, 119, 1451.

49. Mathews, M. R.; Parsons, P. J.; At. Spectrosc. 1993, 14, 41.

50. Vinãs, P.; Campillo, N.; Lopez Garcia, I.; Cordoba, M. H.; Fresenius J. Anal. Chem. 1994, 349, 306.

51. Viñas, P.; Campillo, N.; Lopez Garcia, I.; Cordoba, M. H.; Fresenius J. Anal. Chem. 1995, 351, 695.

52. Lopez Garcia, I.; Viñas, P.; Campillo, N.; Cordoba, M. H.; J. AOAC 1996, $44,836$.

53. Tan, Y-X.; Marshall, W. D.; Blais, J. S.; Analyst 1996, 121, 483.

54. Tan, Y-X.; Blais, J. S.; Marshall, W. D.; Analyst 1996, 121, 1419.

55. Lopez-Garcia, I.; Navarro, E.; Viñas, P.; Cordoba, M. H.; Fresenius J. Anal. Chem. 1997, 357, 642.

56. Viñas, P.; Campillo, N.; Lopez-Garcia, I.; Cordoba, M. H.; Anal. Chim. Acta 1997, 356, 267.

57. Campillo, N.; Viñas, P.; Lopez-Garcia, I.; Cordoba, M. H.; Talanta 1998, 46, 615.

58. Viñas, P.; Pardo-Martinez, P.; Campillo, N.; Cordoba, M. H.; J. AOAC 1999, $82,368$.

59. Volynski, A. B.; Spectrochim. Acta, Part B 1998, 53, 509. 